\title{
Proactive Mitigation of the Revocation Risk for Preemptible Virtualized Resources
}

This paper was downloaded from TechRxiv (https://www.techrxiv.org).

\section{LICENSE}

CC BY 4.0

SUBMISSION DATE / POSTED DATE

$18-08-2021$ / 26-08-2021

\section{CITATION}

Oikonomou, Panagiotis; Kolomvatsos, Kostas; Anagnostopoulos, Christos (2021): Proactive Mitigation of the Revocation Risk for Preemptible Virtualized Resources. TechRxiv. Preprint.

https://doi.org/10.36227/techrxiv.15189558.v1

$\mathrm{DOI}$

10.36227/techrxiv.15189558.v1 


\title{
Proactive Mitigation of the Revocation Risk for Preemptible Virtualized Resources
}

\author{
Panagiotis Oikonomou, Kostas Kolomvatsos and Christos Anagnostopoulos
}

\begin{abstract}
The provision of resources at the Cloud follows two generic models. The first model guarantees the provided resources for the requested time while the second involves unreliable resources with lower price compared to the former scheme but with no guarantees concerning an unexpected revocation due to a high demand. In this paper, we focus on the latter model and propose a scheme that monitors the course of execution of tasks placed at unreliable resources and decides when to store their current progress avoid jeopardizing intermediate outcomes in unexpected revocations. We rely on the principles of Optimal Stopping Theory (OST) to manage multiple tasks and decide for which task and when we have to save its current status. The outcome is a novel checkpointing mechanism fully aligned with the needs of the dynamics of an unreliable environment. The proposed model builds upon the heterogeneity of the available services in the Cloud and concludes a proactive mitigation approach of the revocation risk for unreliable virtualized resources. We present the theoretical basis of our mechanism and describe the solution of the identified problem. The pros and cons of our approach are evaluated through extensive simulations and a set of performance metrics.
\end{abstract}

Index Terms-Cloud Computing, Virtualized resources, Unreliable Resources, Proactive Mitigation, Checkpointing Mechanism, Optimal Stopping Theory, Multi-arm Bandit model

\section{INTRODUCTION}

$\mathrm{T}$ HE diversity of heterogeneous computational resources that Cloud offers has attracted the interest of researchers working in different scientific domains as they can benefit from the variety of the available resources (e.g., CPUs, memory sizes, networking tools/software) to host various processing activities. In Cloud, resources are generally provisioned as Virtual Machines (VMs) claimed in different sizes and pricing models. Two well known pricing models deal with the on-demand and Spot virtualized resources. Ondemand resources have a fixed price for a time interval of usage (e.g., hour or second) while Spot instances are provided at a fraction of the discussed cost but with no guarantees for an uninterrupted usage. Spot resources can be acquired via a bidding policy through an auction-like marker. Greedily, a user claims a Spot resource for the duration that her bid is the maximum. However, the resource is revoked if user's bid price is lower than market price, i.e., another user may acquire the resource. Usually, on-demand resources are selected by users who do not prefer any longterm commitment and for short-term, unpredictable applications that should not be interrupted. On the other hand, Spot resources are best suited for applications that have a flexible duration and deadlines or when there is an urgent need for additional computing capacity. Both pricing models have their own disadvantages, e.g., using exclusively on-demand resources results in enormous monetary costs compared to the Spot pricing model while the invocation of Spot instances has no performance guarantees that may

- P. Oikonomou ans K. Kolomvatsos are with the Department of Informatics and Telecommunications, University of Thessaly, Papasiopoulou 2-4, 35100, Lamia Greece e-mails: \{paikonom, kostasks\}@uth.gr

C. Anagnostopoulos is with the School of Computing Science, University of Glasgow, Lilybank Gardens 17, G12 8RZ, Glasgow UK email:christos.anagnostopoulos@glasgow.ac.uk lead to an increased execution time (due to interruptions) and subsequently to unpredictable costs. We have to notice that in this paper we adopt the concepts of Spot, unreliable and preemptive instances interchangeably.

To mitigate the volatile nature of Spot instances, several mechanisms have been proposed. The naive restart/retry strategy handles failed tasks placed in Spot instances by re-assigning the entire task to another virtualized resource (on-demand or spot) or in the same resource when it will become available again. This mechanism is easy to implement, however, the user must pay for the duration that the Spot instance was utilized even if no progress has been recorded. Another approach uses a mixture of on-demand and Spot instances. Usually, when a task is critical, e.g., belongs to the critical path of a workflow, it is replicated to an ondemand resource to guarantee the deadline constrain while tasks who are dominated by other large computational tasks can be executed in Spot instances without affecting the overall execution time. A checkpointing mechanism saves the progress of a task in discrete time units, thus, in case of a failure (resource revocation), only the remaining part of the task will be executed to the new resource. This mechanism leads to less monetary costs but the adopted scheduler should periodically monitor the queues of resources and backup the progress of tasks even if the possibility of premature termination is relatively low. An abundant checkpointing approach leads to an increased execution overhead. Different checkpointing mechanisms will be discussed in Section 2.

In this work, we elaborate on a new scheduling algorithm that works with the objective to minimize workflow's execution time (makespan) on volatile and other preemptible virtualized instances. The proposed heuristic is based on the Heterogeneous Earliest Finish Time (HEFT) algorithm [1], which is a widely known scheduling model 
for the minimization of workflows makespan. However, the algorithm, along with its extensions (e.g., [2], [3]), does not consider the volatile nature of unreliable resources on scheduling decisions. In this manner, we introduce the uncertainty aware HEFT algorithm (uHEFT) which is capable to migitate the volatile nature of Spot instances using a novel checkpointing mechanism. This mechanism incorporates the principles of the Optimal Stopping Theory (OST) [4] in scheduling decisions about the monitoring of the parallel execution of tasks in the available resources. Through our approach, we seek to identify which is the best time to backup/save/store the progress of tasks execution assigned to unreliable resources under the objective of minimizing the overall monetary cost and the execution time of a workflow. It should be noted that workflows consist of multiple tasks with specific dependencies that should be secured when executed. To the best of our knowledge, this is one of the first efforts that deal with the problem of scheduling scientific workflows using entirely unreliable resources while adopting a time optimized model (i.e., under the control of an OST scheme) to enhance a fault tolerant approach. More specifically, we elaborate on a multi-armed bandit model [5] that tries to detect the optimal strategy related to the selection of tasks for which the status will be stored at every time instance. Our aim is to support a monitoring mechanism upon the parallel execution of multiple tasks placed at the available Spot instances and detect the appropriate time for saving their progress. This way, we will be able to secure their 'smooth' execution without jeopardizing the loss of the intermediate outcomes in case where Spot instances are unexpectedly revoked. The following list reports on the contribution of our work:

- We propose a model that builds upon the heterogeneity of a Cloud environment and the available services. Spot instances can be provisioned in different computing capabilities, also exhibiting a different interruption probability depending on the popularity of each resource;

- We provide a novel checkpointing mechanism to decide on the best time to save the progress of a task when it is executed in unreliable resources. This scheme is integrated as a part of a scheduling algorithm and a monitoring module adopted for the allocation of tasks to the available resources in a way that minimizes the overall execution time and the monetary costs;

- We propose a model that is capable of monitoring the parallel execution of multiple tasks that may be part of a workflow. The proposed monitoring mechanism can detect the task for which we should save its progress in order to avoid the consequences of an unexpected revocation of the Spot instance;

- We perform an extensive experimental evaluation of the proposed model and simulate the execution of real-world scientific workflows. The configuration of the adopted virtualized resources is based on realistic assumptions (AWS).

The remainder of this paper is organized as follows. Section 2 presents the prior work in the domain and exposes the main characteristics of every effort under consideration.
Section 3 discusses the preliminary information about the problem we try to solve and Section 4 presents the proposed mechanism. Section 5 elaborates on the performance of our approach and the presentation of the envisioned experimental evaluation while Section 6 concludes this paper by exposing our future research plans.

\section{Related Work}

Job level Checkpointing. Checkpointing, a mechanism to maintain the reliability of unreliable resources, is introduced in [6]. The authors adopt different real price traces of Amazon's spot instances as input to deliver the probability density function of a failure and conclude to the following dynamic checkpointing strategies: (a) Hour-boundary Checkpointing: it is applied every hour which is actually the lowest granularity of Spot pricing; (b) Rising edge-driven Checkpointing: it is applied when a rising edge occurs i.e., when higher bids are observed compared to the past, (c) Checkpointing with Adaptive Decision: this method analyzes the trade-off between taking or skipping a checkpointing at hourly boundaries. When the expected recovery time of a task with a hour-boundary checkpointing is less than the corresponding recovery time without taking the checkpointing, checkpointing is applied, otherwise it is skipped; and (d) different combinations of the above strategies e.g., checkpointing with adaptive decisions when a rising edge occurs. A main difference between our approach and [6] is that we do not conduct experiments assuming a single task and one unreliable instance as well as when a failure occurs, the task should continue in a Spot resource of the same type. An extension of [6] is presented in [7] where the authors enhance their previous work by integrating a task replication mechanism to minimize the turnaround time of a task executed in a Spot instance. In the migration policy, the user can bid for another type of resource (with different processing capabilities) instead of waiting for the recently used resource. The intuition behind migration is to reduce the recovery time of tasks. Low bid prices may increase exponentially the recovery time as it is more likely an outof-bid event to occur, while migrating a task to another virtualized resource may eliminate task's recovery time with a minor increment in the total cost. In [8], three fault tolerant techniques are presented namely, checkpointing, replication and job duplication. The effect of the three techniques is measured in terms of deadlines and costs, while the pool of the virtualized resources is comprised exclusively by Spot instances distributed in different data centers. The authors also proposed an algorithm which assigns an incoming task to an idle VM with enough time before the time slot (e.g., an hour) finishes. If no such resource exists, the task is allocated to the resource that will become idle sooner than others and simultaneously does not violate the deadline constraints. If this process is not successful, a new Spot instance will be provisioned. The first fault tolerant technique, i.e., checkpointing, is similar to the hour-boundary model presented in [6]. In this scheme, a checkpoint is taken as at every time slot (i.e., an hour). However, instead of saving the state (progress) of a task, the entire VM's state is saved and resumed only if the original bid price allows it. This technique does not consider the provisioning of a 
new resource like the resource migration technique. The migration strategy saves the state of a resource and, in the case of a failure, the remaining work is resumed in (a) a resource of the same type but in a higher price than the previous, (b) a new provisioned resource in the same data center, or (c) to another data center where a suitable resource may be leased for a cheaper price than the previous one. Finally, the job duplication strategies mandates the creation of one replica for each task that is expected to run for more than one time slot (i.e., an hour).

Interval based Checkpointing. In [9], the authors propose a model to calculate the optimal number of checkpoints for Cloud tasks using different probability distributions regarding failure events. The proposed fault-tolerant mechanism does not depend on a particular failure distribution in contrast to works like Young's work [10] or Daly's work [11] which assume that the probability of a failure follows an Exponential distribution. To mitigate the inevitable impact of a failure on the execution time, the authors of [12] propose an estimated interval-based checkpointing (EIC) using the weighted moving average (WMA) and Bollinger Bands. Price and execution time thresholds are defined based on historical traces while their violation triggers the checkpointing mechanism. Bollinger Bands are adopted to predict the bid price in the stock market and estimate the execution time of a task. In [13], a machine learning approach has been designed for predicting the price of Spot instances and facilitate users in bidding. Through the use of the proposed prediction algorithm, a checkpointing mechanism calculates the optimal time intervals to save the progress of each task.

Combining On-demand \& Spot Resources. One of the fundamental problems encountered in the management of large-scale computing systems is to schedule a set of workflows submitted by the users into the available resources. To enhance fault tolerance and increase the reliability of virtualized resources, several approaches adopt a mixture of on-demand and Spot instances on scheduling. For instance, the authors of [14], [15], [16] and [17] study the workflow scheduling problem while the authors of [18], [19], [20] and [21] focus on the bag of tasks scheduling. The concept of Latest Time On-Demand (LTO) is introduced in [14] as the latest time in which on-demand instances must be used to guarantee that a workflow's deadline constraint is not violated. If the difference between LTO and current time is greater than zero (positive slack), tasks are mapped into Spot instances ensuring that the deadline is met while the cost is minimized. The concept of the LTO inspires other works like [15], [16] and [20]. In [14], checkpointing is also applied in three different granularities i.e., every five, ten and thirty minutes. In [15] failures are mitigated using task duplication and resubmission. The goal of the discussed effort is to minimize the execution of the workflow modeled as a Directed Acyclic Graph (DAG) (makespan) within deadlines (similar to [14]). Spot instances can be provisioned in two ways: a) when a positive slack is observed (similar to [14]) or b) when a task is marked for duplication. The scheduler scans the entire set of running instances for one (or more) idle slots that can execute a replica of the task. If no suitable idle slot exists, instead of appending the replica to an already running instance, it is mapped into a new Spot resource. SpotCheck [18] is a system that uses nested virtualization and migration mechanisms to manage a large number of servers supporting the use of both ondemand and Spot resources. In SpotChech, the state of each application is continuously checkpointed in a backup server. When a failure occurs, the stored progress of a task is resumed to an on-demand instance to ensure high availability. In [19], Spot instances are adopted for minimizing the cost of executing a bag of tasks in Cloud. Both, ondemand and Spot instances are used for comparison and the most cost-efficient pricing model is selected. The future cost of a Spot instance is predicted using an Artificial Neural Network (ANN) trained upon historical data collected from previous transactions. In [20], the authors examine the effect of preemptible virtualized resources on the total monetary cost while executing bags of tasks. They propose a framework, named AutoBot, that takes decisions about task placement, checkpointing and migration. The framework also determines the acquisition and release time for ondemand and Spot instances while defining the beneficial bid price. Initially, the bag of tasks is mapped into Spot instances until a critical point $\omega$ which indicates the need to provision-use on-demand instances so as to support deadline guarantees. Since pre-emption may occur at any point in time, three different checkpoint strategies: (a) optimistic: it checkpoints a task only once, just before its local critical point, (b) grace period: it checkpoints when a preemption message is received by the provider, and (c) sliding checkpointing: it guarantees that no progress is lost for a task running on a Spot instance as checkpointing is applied at discrete time units. SpotOn [21] automatically selects the Spot market and the fault tolerance mechanism to mitigate the impact of revocations in unreliable resources. The spot market selection is performed considering the current market price and resources availability, while fault tolerance is achieved in the following axes: (a) reactive migration: checkpointing is applied immediately upon receiving the termination notification message; (b) proactive migration: checkpointing is applied in periodic intervals while the progress of a task is saved only in remote disks; and (c) replicating computation: different Spot markets can be reached (different zones/regions). All the above are translated to a high migration overhead when checkpointing $\mathrm{I} / \mathrm{O}$ intensive applications.

\section{System Model and Problem Formulation}

\subsection{System Model}

We consider the scenario where a scientific workflow, i.e., a set of dependent tasks, should be executed in a set of preemptible virtualized resources offered by a Cloud provider. Definition. A workflow is a set of dependent tasks that solve a scientific problem.

Resources are virtualized (e.g., VMs) and provisioned in a pool configuration prior to the execution of the workflow. The cost of leasing such virtualized resources is bounded between the start time of the first task assigned to it and the completion time of the last task present in the workflow. The cost is rounded up to the nearest billing cycle e.g., hour or second. A workflow application can be modeled as a DAG $G=(T, E)$, where $T$ is the set of tasks and $E$ is the set of edges connecting the vertices. Each edge $e_{i j}$ represents 
data dependencies between tasks $t_{i}$ and $t_{j} . t_{j}$ receives $e_{i j}$ amount of data from its predecessor, i.e., $t_{i}$. For starting the execution of a task $\left(t_{i}\right)$, the following two conditions should hold true: a) all predecessor $\left(\operatorname{pred}\left(t_{i}\right)\right)$ tasks must finish execution and b) all data from $\operatorname{pred}\left(t_{i}\right)$ must be received. $t_{i}$ is characterized by a processing demand parameter $g_{i}$ denoting the number of instructions (i.e., MIPS) that should be executed for its completion. A task is called an entry task $\left(t_{\text {entry }}\right)$ when $\left(\operatorname{pred}\left(t_{i}\right)=\emptyset\right)$. Similarly, a task without any successor $\left(\operatorname{succ}\left(t_{i}\right)=\emptyset\right)$ is called an exit task $\left(t_{\text {exit }}\right)$. If more than one entry tasks exist, then, a pseudo task $t_{\text {pseudo }}$ is inserted to $G$ as the predecessor task of every entry task. No data is transmitted from $t_{\text {pseudo }}$ to any other task. Multiple exit tasks are handled in an analogous manner.

Let $V$ be the set of heterogeneous virtualized resources forming the aforementioned pool configuration. Generally, any virtualized resource can be leased as a reliable or unreliable instance, as discussed, however, in this work, we consider the scenario where only unreliable instances are available. Furthermore, every resource is associated with a processing capability and incurs in a different cost per use. Let $v_{i}$ denote the $i$ th resource, $r_{i}$ be the processing power of $v_{i}$ and $u_{i}$ be the leasing cost of $v_{i}$. Precisely, $r_{i}$ denotes the number of MIPS that can be executed per time unit (i.e., one second) by $v_{i}$. This also incorporates memory speed, disk size and so on and so forth. Each virtualized resource is also associated with a preemption/interruption probability $p_{i}$.

Definition. The preemption/interruption probability $p_{i}$ is the probability of 'loosing' the selected virtualized resource leading to the failure of the corresponding task and the need of a re-execution.

We assume that tasks are 'atomic', i.e., they should be executed from the beginning in case of a failure especially if they have not been checkpointed, otherwise, their execution is resumed from the last saved point. The execution time required to complete the task $t_{i}$ on the resource $v_{j}$ is calculated by Eq. (1).

$$
w_{i j}=\frac{g_{i}}{r_{j}}
$$

We consider that resources are allocated in the same data center, thus, the transfer of inbound data is free. The data center is assumed to have enough resources to schedule the tasks depicted by $G$. A shared global storage system is considered as a data repository, thus, tasks save their outputs and receive their inputs from the same storage system. Since the global storage system is allocated within data center premises, we consider that the data transfer rate between two virtualized resources is constant. Let $T R$ be the data transfer rate and $b_{i j}$ be a binary variable, i.e., $b_{i j}=0$ iff $i=j$ otherwise $b_{i j}=1$. The temporal cost to send data form $t_{i}$ to $t_{j}\left(t_{i}\right.$ is assigned in $v_{i}$ while $t_{j}$ in $v_{j}$ ) is expressed by Eq. (2).

$$
d_{i j}=\frac{e_{i j} b_{i j}}{T R}
$$

$E S T_{t_{i}}^{v_{j}}$ and $E F T_{t_{i}}^{v_{j}}$ are the earliest Execution Start Time (EST) and the earliest Execution Finish Time (EFT) of $t_{i}$ on $v_{j}$. The EST of the entry task is zero. For every other task in $G$, the EST and the EFT are calculated recursively as shown by Eq. (3) and Eq. (4):

$$
E S T_{t_{i}}^{v_{j}}=\max _{t_{z} \in \operatorname{pred}\left(t_{i}\right)}\left\{A F T_{t_{z}}+d_{z i}\right\}
$$

$$
E F T_{t_{i}}^{v_{j}}=w_{i j}+E S T_{t_{i}}^{v_{j}}
$$

When $t_{i}$ is assigned on $v_{j}$ the $E S T_{t_{i}}^{v_{i}}$ is equal to the actual start time $\left(A S T_{t_{i}}\right)$. The same holds true when a task finishes its execution i.e., $A F T_{t_{i}}=E F T_{t_{i}}^{v_{i}}$. The total elapsed time required to execute $G$ (makespan) is the actual finish time of the exit task $\left(t_{\text {exit }}\right)$. Let $N_{i}$ be the set containing all tasks that have been executed by $v_{i}$. Let $t_{k}$ be the $k$ th task assuming a total ordering of them $1 \leq k \leq\left|N_{i}\right|$. Then, the overall execution cost incurred by $v_{i}$ is calculated by Eq. (5), where $\gamma$ is the length of the billing cycle.

$$
c_{i}=u_{i}\left[\frac{A F T_{t_{N}}-A S T_{t_{1}}}{\gamma}\right]
$$

Table 1 summarizes the notation presented in the above discussion.

TABLE 1: Nomenclature

\begin{tabular}{l|l}
\hline \hline Symbol & Meaning \\
\hline$G$ & Workflow application \\
$T$ & Workflow's set of tasks \\
$E$ & Workflow's set of edges \\
$V$ & Set of heterogeneous Cloud resources \\
$t_{i}$ & ith task of $T$ \\
$t_{\text {entry }}$ & The task with no parents \\
$t_{\text {exit }}$ & The task with no children \\
$e_{i j}$ & data dependencies between tasks $t_{i}$ and $t_{j}$ \\
$p r e d\left(t_{i}\right)$ & The parents of $t_{i}$ \\
$s u c c\left(t_{i}\right)$ & The children of $t_{i}$ \\
$g_{i}$ & The number of MIPS instructions for $t_{i}$ \\
$v_{i}$ & $i$ th resource of $V$ \\
$r_{i}$ & Computation capabilities of $v_{i}$ \\
$u_{i}$ & The cost of leasing $v_{i}$ \\
$\gamma$ & Length of the billing cycle \\
$p_{i}$ & Preemption/interruption probability of $v_{i}$ \\
$w_{i j}$ & The execution time of task $t_{i}$ on resource $v_{j}$ \\
$T R$ & Data Transfer Rate \\
$e_{i j}$ & Boolean variable denoting whether $t_{i}$ and $t_{j}$ \\
$d_{i j}$ & are executed in the same resource \\
$E S T_{t_{i}}^{v_{i}}$ & Temporal cost to send data form $t_{i}$ to $t_{j}$ \\
$E F T_{t_{i}}^{t_{i}}$ & The earliest start time of $t_{i}$ on $v_{j}$ \\
$A S T_{t_{i}}$ & The earliest finish time of $t_{i}$ on $v_{j}$ \\
$A F T_{t_{i}}$ & The actual start time of $t_{i}$ \\
$p r o g r e s s_{i}$ & The actual finish time of $t_{i}$ \\
$c c$ & Progress saved for $t_{i}$ \\
$S_{\tau}$ & Checkpoint cost \\
$R()$ & The $\tau$ time slot \\
$\mathbf{d}^{j}$ & Function that delivers the reward of checkpointing \\
$\theta_{j}$ & Historical demand for a task \\
\hline \hline
\end{tabular}

\subsection{The Checkpointing Mechanism}

We consider a monitoring mechanism which is responsible to checkpoint the tasks being executed on the available resources. The proposed model is part of the aforementioned monitoring mechanism triggered when the recorded data indicate that a task (one of the group) should be checkpointed. To checkpoint a task, we have to save its progress/state, i.e., to save task's contents/outcomes from the memory/disk of its dedicated resource and (if needed) transfer it to another resource. Obviously, this procedure introduces extra read/write costs, however, since the cost of storage service is negligible compared to the cost required for execution, we can assume a constant checkpointing cost $c c$. In our model, time is represented by the introduction of $S$ equally sized time slots. Let $S_{\tau}$ be the $\tau$ th time 
slot. If a failure is realized during the execution of any instruction during $S_{\tau}$, we consider that the failure refers to the entire slot. Assume that at every $S_{\tau}$, there are $|V|$ virtualized resources that can host a task. Each one of the $|V|$ resources has a probability of revocation depicted by the variable $p_{j}$. This means that the probability of successfully concluding a task in the $j$ th resource is equal to $\theta_{j}=1-p_{j}$. The successful conclusion of a task leads to a reward $\theta_{j}$ as the execution of the task in the $j$ th resource incurs less cost compared to other Cloud pricing models as it is realized in a Spot instance. For checkpointing the tasks being allocated for execution, we have to decide among two schemes: (i) perform the checkpointing at every $S_{\tau}$ for all the available tasks/resources; (ii) intelligently select the task/resource that will be checkpointed by deciding upon the appropriate time to do that for each task/resource independently. Checkpointing each resource at every $S_{\tau}$ will likely deteriorate workflow's makespan for the following reasons: a) each resource has different task load to finish, b) the probability of revocation is different on each resource, and c) the overall checkpointing overhead which is equal to $|V| \times c c$ could be significantly increased. The second scheme, i.e., independently checkpoint every task/resource, saves time and computational resources if applied at the optimal time slots for each task/resource under the danger of the revocation of the resource before we perform the checkpointing action. Evidently, the decision making of the second scheme is dictated by a model that can efficiently detect the optimal time slot before a revocation event. In that case, for the overall length of the scheduling activity, our monitoring mechanism has to decide which task/resource to checkpoint, how many times and in which order to maximize the desired long-term cumulative reward.

We define the following binary function indicating the failure/revocation of $v_{j}$ :

$$
f\left(v_{j}\right)= \begin{cases}1, & \text { with probability } p_{j} \\ 0, & \text { with probability } \theta_{j}=1-p_{j}\end{cases}
$$

At $S_{\tau}$, we monitor the outcome of a Bernoulli trial with parameter $p_{j} . f_{j}$ depicts the event of revoking a resource during the execution of a task. $p_{j}$ depends on the demand that is observed for resources of the same characteristics like the monitored virtualized asset. Based on $f\left(v_{j}\right)$, we can setup a multi-armed bandit model (MABM) with $|V|$ arms (the resources where the allocated tasks are executed). The MABM is the appropriate mathematical decision scheme for deciding how to optimally perform the envisioned checkpointing in the parallel execution of tasks in $|V|$ resources. The basis of our model is that the decision making is concluded in a sequential order and under randomly evolving conditions which depict the possibility of the revocation for each resource. We also consider that the execution of tasks is treated as independent jobs with the aforementioned binary outcome implemented as Markov rules. These rules depict the activities that could be undertaken when failures or successes are observed. Obviously, the most critical activity is the management of failures and the detection of the appropriate time to perform the checkpointing for each task/resource. Our 'reasoning' is dictated by two parts: (i) to correctly detect the appropriate tasks to initiate the checkpointing action, i.e., the exploration or learning phase; (ii) to start the checkpointing action and secure that the current state of the corresponding task is secured, i.e., the exploitation or earning phase.

The 'behaviour' of every task/resource (i.e., the arms of our bandit in the MABM) is depicted by the random variable $Y_{j} \sim \operatorname{Bernoulli}\left(p_{j}\right)$ with unknown success probability $p_{j}$. We consider that the 'success' of our Bernoulli trial is to checkpoint the monitored resource at the time just before it is revoked. We do not want to checkpoint a resource many slots before because we can lose the intermediate results. The optimal time for checkpointing a resource is when it is planned to be revoked by the provider. If checkpointing a resource just before it is revoked, we gain a specific reward dictated by the following function:

$$
R\left(v_{j}, S_{\tau}\right)= \begin{cases}\alpha *\left(\left(S_{\tau}-A S T_{i}\right)-c c\right), & \text { if } f\left(v_{j}\right)=1 \\ 0, & \text { otherwise }\end{cases}
$$

where $\alpha$ is a constant. The closer the $S_{\tau}$ is to the $E F T_{j}$, the highest the reward becomes. This is because only a few slots remain for the conclusion of the execution and we checkpoint the task before the revocation action. The value $\left(S_{\tau}-A S T_{i}\right)$ expresses the current executed duration of $t_{i}$ i.e, the total amount of progress than can be saved. Checkpointing $v_{j}$ is not beneficial (zero reward) when the checkpoint overhead $(S \tau+c c)$ exceeds the estimated finish time $\left(E F T_{i}\right)$ of the currently executed task $t_{i}$ will finish its execution sooner (before checkpointing is concluded). The expected reward at $S_{\tau}$ is calculated by $\mathrm{E}(R)=p_{j} * \alpha *\left(\left(S_{\tau}-A S T_{i}\right)-c c\right)$. Evidently, $p_{j}$ is unknown and affected by the demand that users exhibit about the specific type of resource. Hence, we consider that we also monitor the demand for each type of resources and rely on the last $W$ observations. Let the vector of the latest demand observations for the $j$ th resource as dictated by the random variable $D^{j}$ be $\mathbf{d}^{j}=\left[d_{1}^{j}, d_{2}^{j}, d_{3}^{j}, \ldots, d_{W}^{j}\right] . p_{j}$ can be easily estimated through the use of a kernel method for exposing the probability density function (pdf) of $D^{j}$ and the corresponding cumulative distribution function (cdf). Actually, $p_{j}$ is represented by the probability of having the demand for a resource over a pre-defined threshold $\xi$, i.e., $p_{j}=P\left(D^{j}>\xi\right)=1-P\left(D^{j} \leq \xi\right)=1-\mathbf{F}_{D^{j}}(\xi)$ where $\mathbf{F}_{D^{j}}(\cdot)$ is the cdf of the random variable $D^{j} \cdot p_{j}$ depicts the success probability of our Bernoulli trial and depends on the pdf of the distribution representing the demand for the specific virtualized resources. The estimation of the pdf of $D^{j}$ can be realized as studied in [22]. We apply the widely known Kernel Density Estimator (KDE) [23] to derive the distribution of $D^{j}$ and the corresponding pdf.

The statistical learning process is applied on top of $\mathbf{d}^{j}$ which represent the historical demand values. We estimate the pdf $\hat{f}\left(\mathbf{d}^{j}\right)$ through the KDE as follows: $\hat{f}\left(\mathbf{d}^{j}\right)=$ $\frac{1}{W \cdot h} \sum_{i=1}^{W} K\left(\frac{x-d_{i}^{j}}{h}\right) . h>0$ represents the bandwidth of the adopted Kernel function $K(\cdot)$ that is is symmetric and integrates to unity. For $K(\cdot)$, we adopt the Gaussian kernel, $\left(K(z)=\frac{1}{\sqrt{2 \pi}} \exp \left(-\frac{1}{2} z^{2}\right)\right)$. The realization of $p_{j}=$ $1-\mathbf{F}_{D^{j}}(\xi)$ involves the definition of the cdf of $\hat{f}\left(\mathbf{d}^{j}\right)$, i.e., $p_{j}=1-\int_{-\infty}^{\xi} \hat{f}(x) d x$. Based on the properties of integrals, we get: $p_{j}=1-\frac{1}{W \cdot h} \sum_{i=1}^{W} \int_{-\infty}^{\xi} K\left(\frac{x-d_{i}}{h}\right) d x$. We have to 
notice that the $\mathrm{KDE}$ is a mixture density depicted by $\hat{f}(\cdot)$; the cdf of the mixture is also the mixture of cdfs. Hence, we can get $\mathbf{F}_{D^{j}}(\cdot)=\Phi\left(\frac{x-d_{i}}{h}\right)$. However, for 'translating' the new variable defined by the KDE model to the standard Normal distribution we get: $P\left(\frac{x-d_{i}}{h} \leq \xi\right)=P\left(x \leq h \cdot \xi+d_{i}\right)=$ $P\left(\frac{x-\mu^{j}}{\sigma^{j}} \leq \frac{h \cdot \xi+d_{i}-\mu^{j}}{\sigma^{j}}\right)$. Based on the above description, we finally get: $p_{j}=1-\frac{1}{W \cdot h} \sum_{i=1}^{W} \Phi\left(\frac{h \cdot \xi+d_{i}-\mu^{j}}{\sigma^{j}}\right)$. As it is difficult to find a closed form for the cdf of the Gaussian distribution, many research efforts study various approximations on it [24]. We rely on the approximation proposed by [25], i.e., $\Phi(z) \approx 0.5+0.5 \sqrt{1-e^{-\sqrt{\frac{\pi}{8}} z^{2}}}$.

Research Challenge: Select the appropriate $S_{\tau}$ to checkpoint the task allocated at $v_{j}$ in order to maximize the total reward $\sum_{\forall j, \forall \tau} R\left(v_{j}, S_{\tau}\right)$ which is referred as the the regret of the model, i.e., the expected difference between the reward associated with the optimal strategy (checkpointing at the revocation time).

\section{The Uncertainty aware Heterogeneous EARLIEST FINISH TIME ALGORITHM (UHEFT)}

For our scheduling scenario, decisions for each task and for each checkpoint are made at runtime i.e., when a task is ready for execution, or when a failure event is observed. For this reason, we focus on discrete events and decompose our problem into a set of logically separate processes that autonomously progress over time. We consider that each event is a tuple of four elements: $E=\langle$ type,time, vm,task $\rangle$, i.e., type describes the type of the event, time is the time instance where the event is observed, and $v m$ and task denote the resource and task that event $E$ is associated with. Table 2 summarizes four different types of events adopted in our model and the corresponding permissible values for the elements time, vm and task.

TABLE 2: Type of events

\begin{tabular}{cccc}
\hline Type & time & $v m$ & task \\
\hline \hline EXECUTE & any & any & any \\
FINISH & any & any & any \\
REVOKE & any & any & null \\
CHECKPOINT & every $S \tau$ & all & null \\
\hline
\end{tabular}

Events of type EXECUTE indicate that a task is retrieved by the ready queue (task is in ready state) in order to be assigned into one of the available resources. This event is triggered when the conditions for running a task are met i.e, all predecessor tasks are finished and the required input data have been received. Events of type FINISH express that a task from the workflow finished its execution into a specific resource. An event of type $R E V O K E$ indicates that a VM (third element in tuple) will be terminated and returned back to the provider. This type of events can occur at any time instance. $C H E C K P O I N T$ events indicate that a resource will be checkpointed. At predefined intervals, CHECKPOINT events are triggered by the monitoring mechanism as explained in Section 3.2. In consequence, a selected (by the proposed model) resource will be checkpointed. An event of type FINISH is generated when a task finishes its execution. It is also released at time $\tau=E F T_{t_{i}}^{v_{\text {best }}}$ which is the earliest finish time for $t_{i}$ as proposed by the HEFT algorithm [1]. Algorithm 1 and listeners 1, 2, 3 and 4 describe the operation of the proposed uHEFT algorithm.

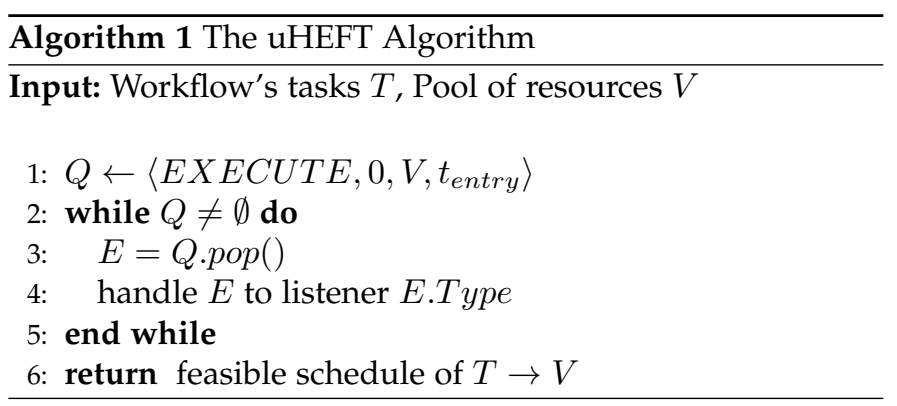

We assume a priority queue $Q$ that contains events of all types (EXECUTE, FINISH, REVOKE and $C H E C K P O I N T$ ). At first (time 0), we enqueue in $Q$ an event of type EXECUTE for the entry task of $G$. The algorithm is completed when $Q$ becomes empty i.e., all tasks are assigned into the available resources and subsequently finished their execution while every REVOKE/CHECKPOINT event is handled by the monitoring mechanism.

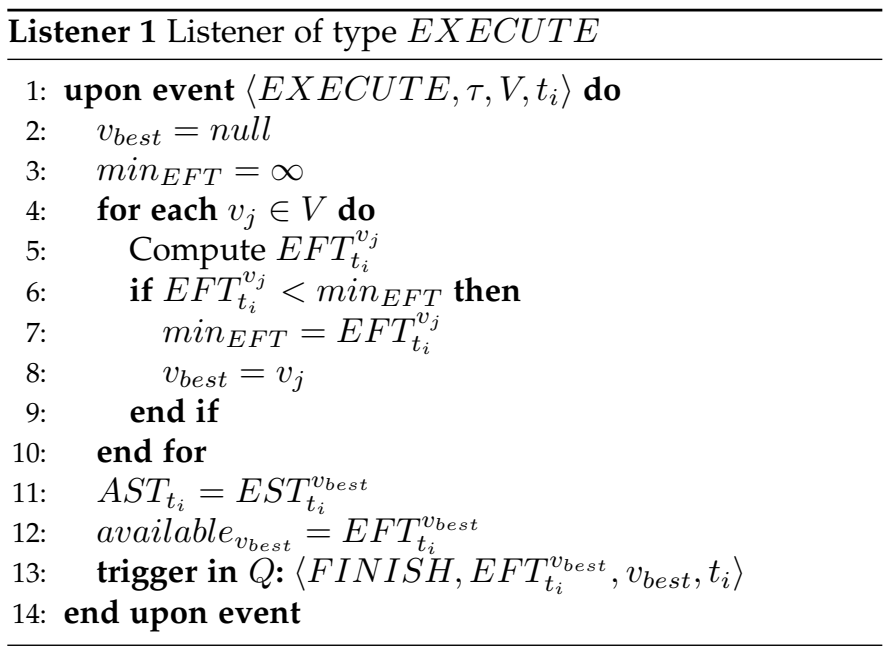

Listener 1 is dedicated to handle events of type $E X E C U T E$. For every available resource $v_{j}$, we estimate the finish time of task $t_{i}$ using eq. (4) (line 5). Finally, $t_{i}$ is assigned to the resource $\left(v_{\text {best }}\right)$ that results in the minimum EFT (lines 11,12). In line 13 , an event of type FINISH is enqueued into $Q$ to signal the fulfilment of task $t_{i}$.

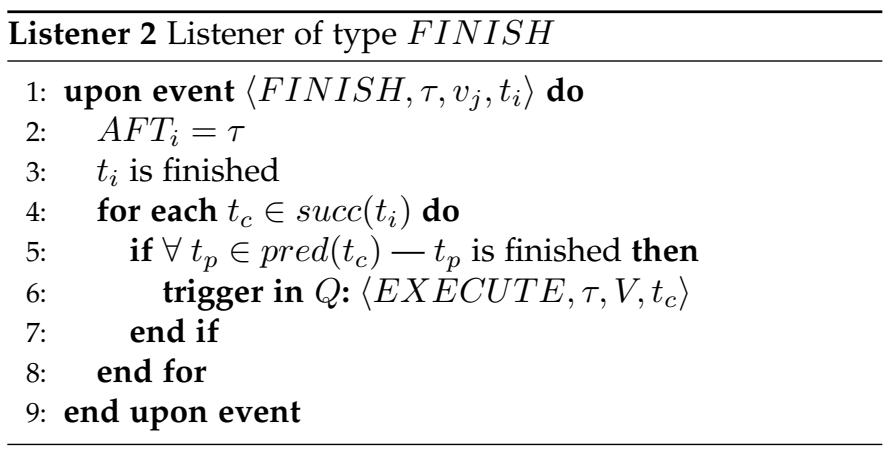

Listener 2 is triggered upon the completion of a task $\left(t_{i}\right)$. Then, for every successor task of $t_{i}$ (line 4 ) we generate a 
new EXECUTE event (line 6) if and only if all predecessor tasks have concluded their execution (line 5).



Listener 3 is dedicated to handle events of type $R E V O K E$ at time $\tau$. An event of this type signals the termination of a resource $\left(v_{j}\right)$. However, as one or more tasks may be already assigned for execution in $v_{j}$ at time later than $\tau$ (line 2) i.e., a task is in waiting state as its execution did not started yet, before the termination of $v_{j}$, we have to resubmit/retry those tasks back to the scheduler in order to execute them into a new resource. Since $v_{j}$ will not be available after time $\tau$ every FINISH event that denotes the completion of a task into $v_{j}$ has to be removed from $Q$ (line 2). For the resumption phase (line 4), an $E X E C U T E$ event is enqueued to $Q$ for every task that did not managed to start execution in $v_{j}$. Regarding lines $6-9, t_{\text {rev }}$ denote the task (if any) that at time $\tau$ is executed in $v_{j}$. This task will be resubmitted back to scheduler too, however, if the checkpointing mechanism (listener 4) has already checkpoint the progress of $t_{r e v}$, then, its computational requirements are decreased according to the amount of progress saved $\left(\right.$ saved $_{\text {rev }}$ ). In other words, only a part of $t_{\text {rev }}$ will be re-executed to another resource (line 7) while the task can be checkpointed again in another resource. Each unfinished task should be resubmitted for execution in another resource at line 10.

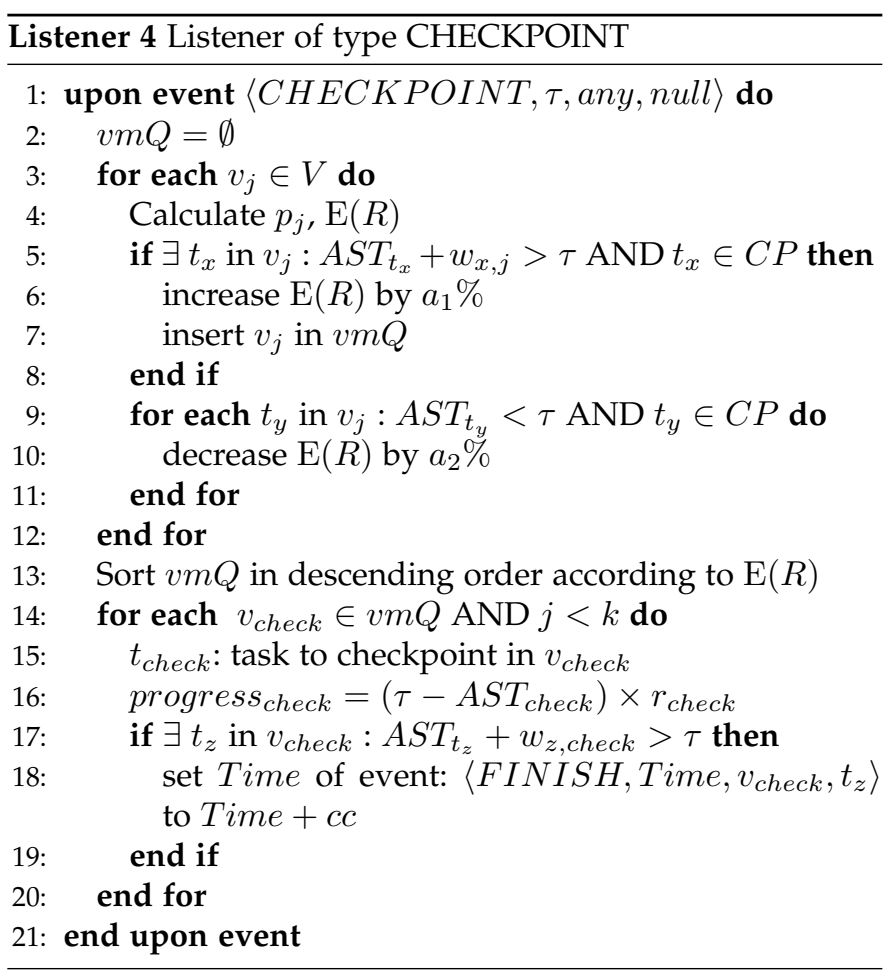

In Listener 4 a checkpoint event is performed at every $S_{\tau}$ time slot. After calculating the probability $p_{j}$, we can estimate the reward of our multi-armed bandit model as follows. We rely on the Follow Perturbed Leader (FPL) algorithm [26] to discern the 'arms', i..e., the virtualized resources that will be checkpointed. The FPL model applies a random noise on the reward of the checkpointing activity for each virtualized resource based on an exponential function $H_{j}\left(S_{\tau}\right)=e^{\zeta}$ with $\zeta \in \mathrm{R}^{+}$. The final reward for each resource is given by $R_{j}^{F}=\mathrm{E}\left(R_{j}\right)+H_{j}$. The final reward is sorted in a descending order and the top$k$ resources are checkpointed. It should be noted that based on this approach, we try to apply the necessary exploration and exploitation phases of the envisioned setting. When a resource exhibits a very high demand, the corresponding probability will be increased causing checkpointing events as our algorithm indicates.

Tasks that, at time $\tau$, are executed and, at the same time, belong to the critical path $(\mathrm{CP})$ of the workflow are rewarded by a percentage of $a_{1}$. We apply this technique as in workflow scheduling the main objective is to reduce the overall execution time of a workflow i.e., the makespan. This objective is controlled by the tasks that belong to the $\mathrm{CP}$, i.e., the longest path existing in workflows that connects entry and exit nodes. On the other hand, we penalize the expected reward by a percentage of $a_{2}\left(a_{2} \ll a_{1}\right)$ for every task that is scheduled to start execution in the specific VM in time higher than $\tau$. Let $t_{\text {check }}$ denote the task that will be checkpointed on a selected resource $v_{\text {check }}$. The total execution time saved after applying the checkpointing activity on $t_{\text {check }}$ is $\tau-A S T_{\text {check }}$, i.e, the number of MIPS for $t_{\text {check }}$ is progress $s_{\text {check }}=\left(\tau-A S T_{\text {check }}\right) \times r_{\text {check }}$, where $r_{c h e c k}$ denotes the computation capabilities of $v_{\text {check }}$. Since checkpointing incurs an overhead of $c c$, both $t_{\text {check }}$ as well the rest of the tasks that are in the waiting state should slow down by $t c$ time units. 


\section{Experimental EVALUATion}

\subsection{Simulation setup}

Workflow Applications. We report on the experimental evaluation of the proposed model relying on six (6) workflow applications as depicted by [27], [28]. The number of tasks, the execution time of each task as well the amount of data transferred between them are reported in a 'Directed Acyclic Graph in XML' (DAX) format. Workflows include Montage, CyberShake, LIGO, SIPHT, Genome and Epigenomics which are extensively adopted for experimentation in the relevant literature. The discussed workflows 'cover' all the basic execution patterns such as pipelining, process, data aggregation, data distribution and data redistribution. Each workflow contains 100 tasks.

Virtualized Resources. We consider a Cloud model with a single data center offering VMs of different CPU speeds and prices. For each experiment, we consider multiple unreliable resources with their characteristics being generated upon the Amazon EC2 platform. The average bandwidth between the storage system (S3) and VMs is set to $20 \mathrm{Mbps}$ which is the approximate average bandwidth provided by Amazon services [29]. To measure the performance fluctuations of the adopted VMs, we follow a similar approach as the one presented in [30], [31]. The performance of VMs varies up to $19 \%$ based on a normal distribution with a mean of $9.5 \%$ and standard deviation of $5 \%$. The bootup/startup time for each VM (provisioning time) is set to 96.9 seconds [32].

The Interruption-Checkpointing Model. In our experimental evaluation, we consider that interruptions may occur at any slot $\left(S_{\tau}\right)$ during the execution of tasks in any unreliable resource. After an interruption, the corresponding $\mathrm{VM}$ is requested from the provider (provisioning and deprovisioning costs are considered). We also assume a fixed checkpointing overhead similar to [6], [7] and [12]. The demand for the resources is randomly produced in the unity interval adopting a Uniform distribution in order to simulate a very dynamic environment with heavy fluctuations. Actually, the random generation of the demand consists of the worst case scenario for our experimentation.

Performance Metrics. To evaluate the performance of the proposed model we adopt the following set of metrics: (i) the regret of our multi-armed bandit model $(\rho)$ compared to the theoretical optimal strategy, (ii) the number of checkpoints $C T$ and (iii) the number of retried tasks $R T$. All metrics where measured against an optimal strategy $(O P T)$ in which a checkpoint is applied just before a revocation event is observed. To be more precise, $\rho$ is measured as the ratio $\frac{M_{u H E F T}}{M_{O P T}} \cdot M_{u H E F T}$ denotes workflow's makespan after applying the proposed checkpointing scheme while $M_{O P T}$ denotes workflow's makespan after applying the optimal strategy $(O P T)$. Similarly, $C T$ and $R T$ are measured using the latter ratio. In total, we conduct 100 iterations for each experiment and get the mean of the aforementioned metrics. For each experimental scenario, we keep $k=1$ to checkpoint at most one VM per time slot. Trade-offs for different $k$ values are revealed in Figs. 13 and 14. Experiments are conducted on a Linux server with two 6-core Intel Xeon E5-2630 CPUs running at $2.3 \mathrm{GHz}$.

\subsection{Performance Assessment}

Initial, we perform a set of simulations for different checkpoint costs (cc) and illustrate its effects on $\rho$ (Fig. 1), $C T$ (Fig. 3) and $R T$ (Fig. 5). $c c$ varies from zero (no checkpoint cost) to 20 (high checkpoint cost). Since workflows have different structure, size and computational demand we set each $c c$ value as the percentage of workflow's mean computation cost. We also keep $W=10, V=8$ and $\xi=0.5$. As we observe from Fig 1, in most cases the mean $\rho$ is around twice the regret delivered by the $O P T$ strategy which is quite encouraging for our approach. Recall that $O P T$ is theoretical optimal strategy which is assumed to know beforehand the precise actual time when a revocation event will happen. As expected, as the cost for checkpoint increases the performance of the proposed method decreases. This reflects the fact that more tasks will be retried (Fig. 5 and subsequently will be checkpointed again (Fig. 3) since resources remain occupied for a longer period of time. Additionally, the proposed approach is characterized by the intense need to deal with the uncertainty around the revocation events, thus, it results a high $\rho$ for a high $c c$. Regarding the SIPHT workflow, in order to achieve the same good performance as the montage workflow, it requires about two times more checkpoints compared to the $O P T$ strategy. This can be explained as Sipht is a balanced workflow with both high I/O activities and computational intensive tasks. Concerning $C T$ and $R T$, we observe a similar behaviour, however, for some workflows (e.g., Montage, Ligo), our model exhibits the best possible performance close to the $O P T$ strategy.

In Figs. 2, 4 and 6, we plot the performance of the uHEFT for various $W$ (window size) realizations. We also keep $c c=10, V=8$ and $\xi=0.5$. We can observe that for $W=10$ all workflows (except SIPHT) are concluded faster than the other experimental scenarios for various $W$ values. This stems from the fact that for large $W$ values $(W>10)$, the demand for a resource may significantly vary in different time units while for low $W$ values $(W<10)$, we do not have enough information to feed the KDE scheme and apply the statistical learning process. The variety of the adopted workflows leads to a variety of the outcomes for $\rho$, $C T, R T$. There are workflows that are positively affected by $W$ (a high $W$ leads to outcomes close to the $O P T$ strategy) while others exhibit the opposite results. In general, the fluctuations of results for the aforementioned metrics could not be judged as significant.

In Figs. 7, 9 and 11, we present the performance of the proposed algorithm when the number of VMs used varies from 2, 4, 8 and 16. We also keep $c c=10, W=10$, and $\xi=0.5$. When only 2 VMs are utilized, uHEFT achieves a regret of 1.5 compared to $O P T$ for all workflows but it requires 3 times more checkpoints. This stems from the fact that each workflow consists of 100 tasks, thus, using only two resources will increase the overall execution time and subsequently the total number of checkpoints. As the number of VMs increases, uHEFT exploits the parallelism degree of each workflow and maps as many tasks as possible into the available VMs. For this reason the $C T$ metric decreases as the number of VMs increases leading to the same number of checkpoints (for $8 \mathrm{VMs}$ ) compared to $O P T$ with a mean 
regret equal to 1.7. The over-provisioning of VMs (16 VMs) deteriorates the performance of the proposed algorithm as the vast majority of them remain under-utilized in most of the experimentation time. Concerning the $C T \& R T$ metrics, we observe opposite directions in the oucomes, i.e., $C T$ is decreased as the number of virtualized resources increases while at the same time $R T$ is increased.

Finally, we record the performance of uHEFT as the threshold $\xi$ increases from 0.2 to 0.8 . Figs. 8,10 and 12 present the performance in terms of $\rho, C T$ and $R T$, respectively. We also keep $c c=10, W=10$ and $V=8 . \rho$ is observed to be decreased as $\xi$ increases; the same stands for $R T$. $C T$ is increased for high values of $\xi$. Recall that $\xi$ depicts the demand threshold over which a task can be considered as very popular. A high demand leads to the best possible performance having our model to be very close to the $O P T$ strategy in terms of $\rho$. This means that we gain high reward for popular tasks and the increased number of checkpoints (depicted by $C T$ ), we are able to detect the best time instance to save the current state of the executed tasks.

In Figs. 14 and 13, we plot $\rho$ and $C T$ for different combinations of $k$ and $c c$ while we set $W=10, V=8$ and $\xi=0.5$. The aforementioned metrics where evaluated for the Epigenomics workflow that has the worst performance according to Fig. 1. For $k=1$ (only one task is selected for checkpointing), we can observe that any performance difference is rather low regarding the cost for checkpointing. For $c c=0$ and as $k$ increases, we can observe that $\rho$ tends to one (it exhibits the same performance as $O P T$ ) which is reasonable as by checkpointing more VMs (they are all checkpointed when $k=8$ ) is more likely to save the entire progress of every task. For $c c=10$, the performance of the uHEFT is stabilized when more than half of the available VMs are checkpointed while for $k=8$, we observe a minor increment in $\rho$. Nonetheless, $C T$ increases from 3 to 4 times more checkpointing events compare to the $O P T$. The upper line $(c c=20)$ clearly presents the drawbacks when checkpointing the vast majority of VMs for a large checkpoint cost. To achieve a good performance, only a small fraction of the available VMs should be considered for checkpointing events.

\section{Conclusions \& Future Work}

The provision of intelligent and high performance applications in Cloud involve the efficient management of application workflows. Every workflow may consist of a number of tasks, thus, a scheduler can undertake the responsibility of allocating them into available virtualized resources and administrate their outcomes. In this work, we focus on the management of unreliable resources, i.e., virtualized instances that can be revoked by the provider at any time. This means that we have to take into consideration the current state of tasks, save their intermediate results and continue the execution when a new instance is available. A significant research challenge is related to the detection of the appropriate time to checkpoint the executed tasks and store their state before any revocation action takes place. We rely on the principles of Optimal Stopping Theory and, more specifically, on a multi-armed bandit scheme. We model the problem under consideration and provide the theoretical background that is 'fed' into a new algorithm. This algorithm tries to be uncertainty aware, i.e., to identify resources that may be revoked, thus, to save the state of the corresponding tasks. We provide the outcome of an extensive experimentation and compare the proposed approach with the theoretical optimal performance boundary that assumes full knowledge on future revocation events. Our evaluation exhibits that the proposed model can approach the results provided by the optimal model exhibiting a solution that can be easily incorporated into a monitoring mechanism. In the first place of our future research agenda is the adoption of a fuzzy based inference scheme that will adopt a set of rules upon a number of parameters that are critical for decision making in the domain under consideration.

\section{REFERENCES}

[1] H. Topcuoglu, S. Hariri, and M. Wu, "Performance-effective and low-complexity task scheduling for heterogeneous computing," IEEE Transactions on Parallel and Distributed Systems, vol. 13, no. 3, pp. 260-274, 2002.

[2] Y. Samadi, M. Zbakh, and C. Tadonki, "E-heft: Enhancement heterogeneous earliest finish time algorithm for task scheduling based on load balancing in cloud computing," in HPCS, pp. 601609, 2018.

[3] K. R. Shetti, S. A. Fahmy, and T. Bretschneider, "Optimization of the heft algorithm for a cpu-gpu environment," in 2013 International Conference on Parallel and Distributed Computing, Applications and Technologies, pp. 212-218, 2013.

[4] T. P. Hill, "Knowing when to stop," American Scientist, vol. 97, 2009.

[5] P. Auer, N. Cesa-Bianchi, and P. Fischer, "Finite-time analysis of the multiarmed bandit problem," Machine Learning, vol. 47, 2002.

[6] S. Yi, D. Kondo, and A. Andrzejak, "Reducing costs of spot instances via checkpointing in the amazon elastic compute cloud," in 2010 IEEE 3rd International Conference on Cloud Computing, pp. 236243, IEEE, 2010.

[7] S. Yi, A. Andrzejak, and D. Kondo, "Monetary cost-aware checkpointing and migration on amazon cloud spot instances," IEEE Transactions on Services Computing, vol. 5, no. 4, pp. 512-524, 2011.

[8] W. Voorsluys and R. Buyya, "Reliable provisioning of spot instances for compute-intensive applications," in 2012 IEEE 26th International Conference on Advanced Information Networking and Applications, pp. 542-549, IEEE, 2012.

[9] S. Di, Y. Robert, F. Vivien, D. Kondo, C.-L. Wang, and F. Cappello, "Optimization of cloud task processing with checkpoint-restart mechanism," in Proceedings of the International Conference on High Performance Computing, Networking, Storage and Analysis, pp. 1-12, 2013.

[10] J. W. Young, "A first order approximation to the optimum checkpoint interval," Communications of the ACM, vol. 17, no. 9, pp. 530531, 1974.

[11] J. T. Daly, "A higher order estimate of the optimum checkpoint interval for restart dumps," Future generation computer systems, vol. 22, no. 3, pp. 303-312, 2006.

[12] D. Jung, J. Lim, H. Yu, and T. Suh, "Estimated interval-based checkpointing (eic) on spot instances in cloud computing," Journal of Applied Mathematics, vol. 2014, 2014.

[13] A. K. Mishra, D. K. Yadav, Y. Kumar, and N. Jain, "Improving reliability and reducing cost of task execution on preemptible vm instances using machine learning approach," The Journal of Supercomputing, vol. 75, no. 4, pp. 2149-2180, 2019.

[14] D. Poola, K. Ramamohanarao, and R. Buyya, "Fault-tolerant workflow scheduling using spot instances on clouds," Procedia Computer Science, vol. 29, pp. 523-533, 2014.

[15] D. Poola, K. Ramamohanarao, and R. Buyya, "Enhancing reliability of workflow execution using task replication and spot instances," ACM Transactions on Autonomous and Adaptive Systems (TAAS), vol. 10, no. 4, pp. 1-21, 2016.

[16] M. Suguna, D. Prakash, D. Y. Thangam, and G. Shobana, "Heuristic task workflow scheduling in cloud using spot and on-demand instances," Journal of Computational and Theoretical Nanoscience, vol. 15, no. 8, pp. 2640-2644, 2018. 


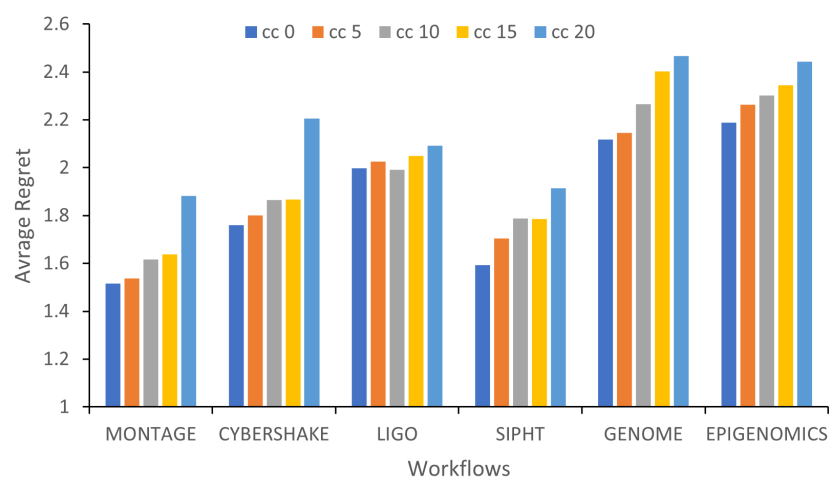

Fig. 1: Regret $\rho$ for different $c c$ values

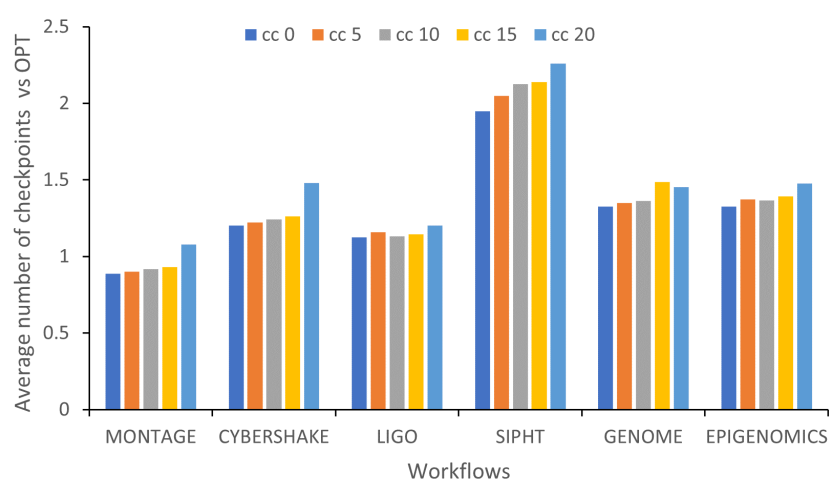

Fig. 3: $C T$ for different $c c$ values

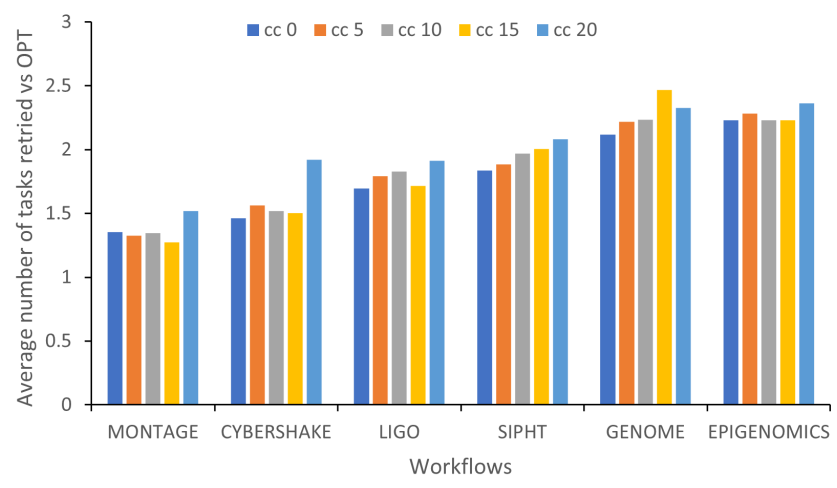

Fig. 5: $R T$ for different $c c$ values

[17] L. Chen, X. Li, and R. Ruiz, "Cloud workflow scheduling with on-demand and spot block instances," in 2017 IEEE 21st International Conference on Computer Supported Cooperative Work in Design (CSCWD), pp. 451-456, IEEE, 2017.

[18] P. Sharma, S. Lee, T. Guo, D. Irwin, and P. Shenoy, "Spotcheck: Designing a derivative iaas cloud on the spot market," in Proceedings of the Tenth European Conference on Computer Systems, pp. 1-15, 2015.

[19] S. G. Domanal and G. R. M. Reddy, "An efficient cost optimized scheduling for spot instances in heterogeneous cloud environment," Future Generation Computer Systems, vol. 84, pp. 11-21, 2018.

[20] P. Varshney and Y. Simmhan, "Autobot: Resilient and costeffective scheduling of a bag of tasks on spot vms," IEEE Transactions on Parallel and Distributed Systems, vol. 30, no. 7, pp. 15121527, 2018.

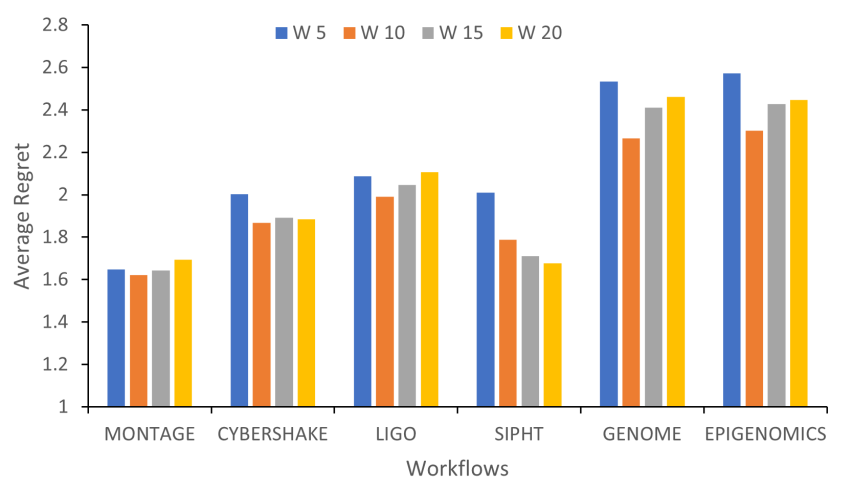

Fig. 2: Regret $\rho$ for different $W$ values



Fig. 4: $C T$ for different $W$ values

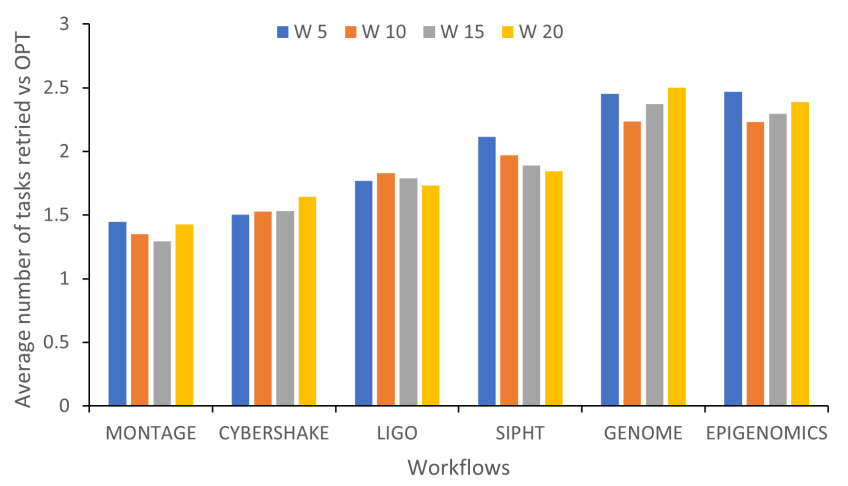

Fig. 6: $R T$ for different $W$ values

[21] S. Subramanya, T. Guo, P. Sharma, D. Irwin, and P. Shenoy, "Spoton: a batch computing service for the spot market," in Proceedings of the sixth ACM symposium on cloud computing, pp. 329-341, 2015.

[22] A. Karanika, P. Oikonomou, K. Kolomvatsos, and T. Loukopoulos, "A demand-driven, proactive tasks management model at the edge," in IEEE International Conference on Fuzzy Systems (FUZZIEEE), IEEE, 2020.

[23] M. P. Wand and M. C. Jones, "Kernel smoothing," Chapman and Hall, 1995.

[24] Y. R. and B. N. K., "Approximations to standard normal distribution function," International Journal of Scientific and Engineering Research, vol. 6, no. 4, 2015.

[25] A. K. M. and A. M. T., "A note on approximating the normal distribution function," Applied Mathematical Sciences, vol. 2, no. 9 , pp. 425-429, 1995. 


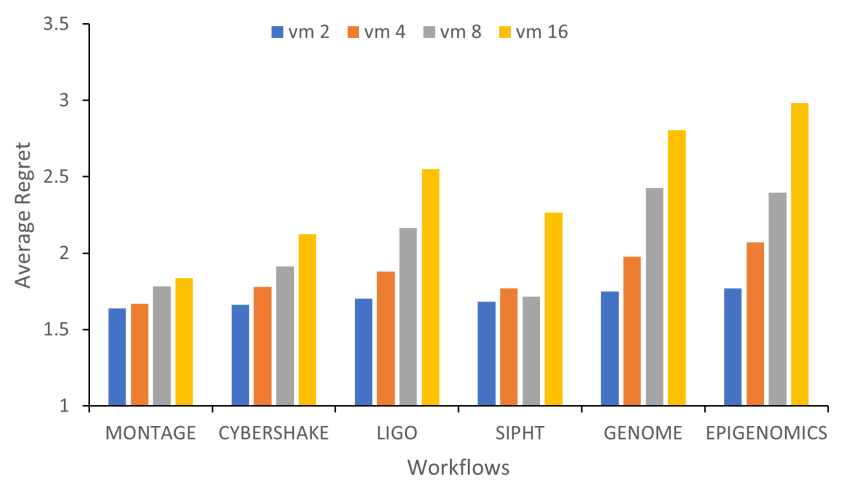

Fig. 7: Regret $\rho$ for different $V$ values

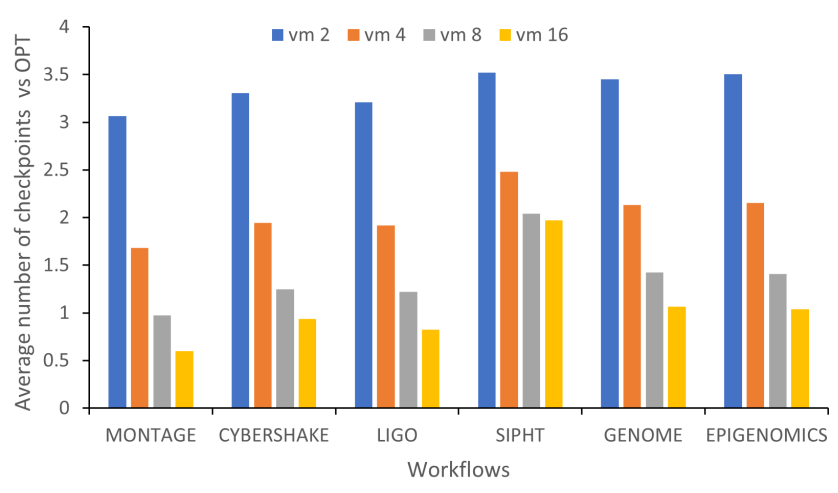

Fig. 9: $C T$ for different $V$ values

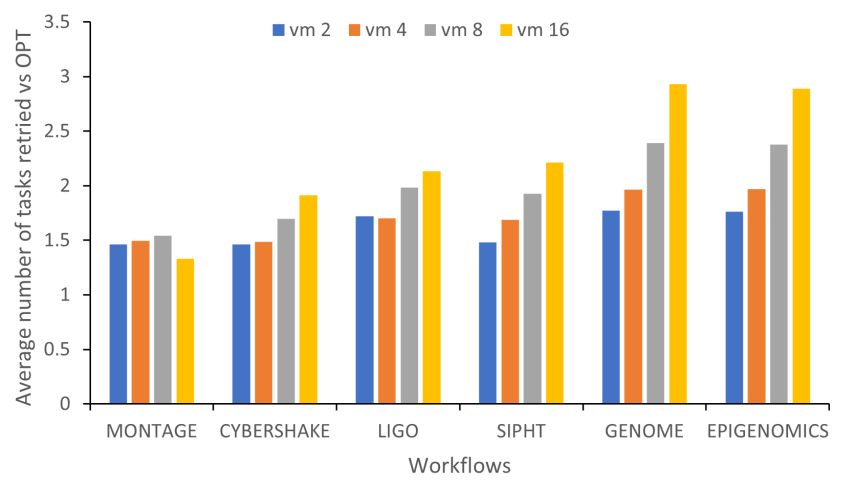

Fig. 11: $R T$ for different $V$ values

[26] V. V. V., "The follow perturbed leader algorithm protected from unbounded one-step losses," in 20th International Conference on Algorithmic Learning Theory, 2009.

[27] G. Juve, A. Chervenak, E. Deelman, S. Bharathi, G. Mehta, and K. Vahi, "Characterizing and profiling scientific workflows," $\mathrm{Fu}$ ture Generation Computer Systems, vol. 29, no. 3, pp. 682-692, 2013.

[28] S. Bharathi, A. Chervenak, E. Deelman, G. Mehta, M.-H. Su, and K. Vahi, "Characterization of scientific workflows," in 3rd workshop on workflows in support of large-scale science, pp. 1-10, IEEE, 2008.

[29] M. R. Palankar, A. Iamnitchi, M. Ripeanu, and S. Garfinkel, "Amazon s3 for science grids: a viable solution?," in International workshop on Data-aware distributed computing, pp. 55-64, 2008.

[30] J. Schad, J. Dittrich, and J.-A. Quiané-Ruiz, "Runtime measurements in the cloud: observing, analyzing, and reducing variance," Proceedings of the VLDB Endowment, vol. 3, no. 1-2, pp. 460-471,



Fig. 8: Regret $\rho$ for different $\xi$ values

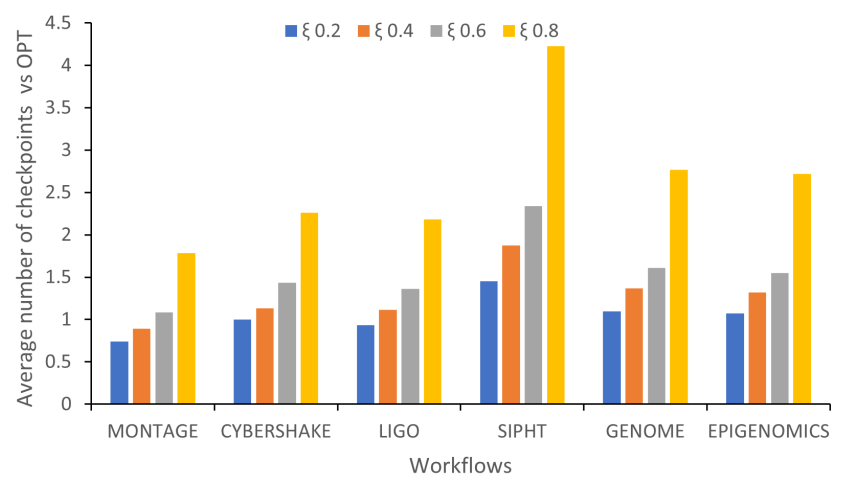

Fig. 10: $C T$ for different $\xi$ values

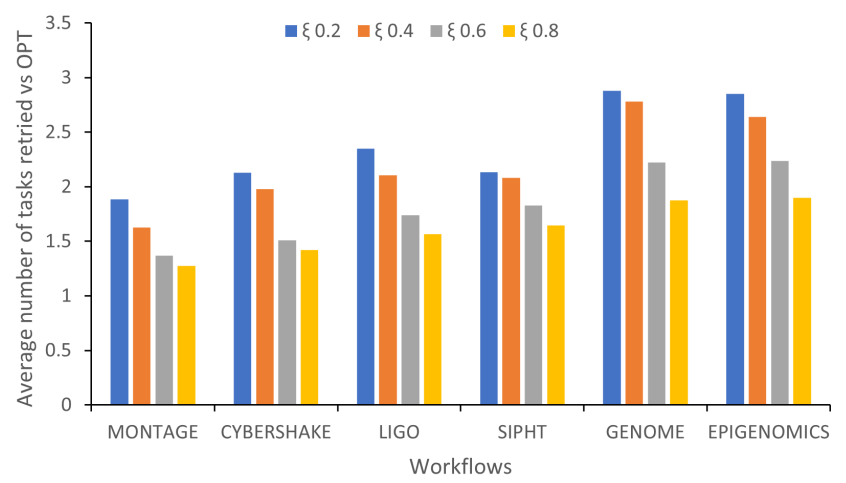

Fig. 12: $R T$ for different $\xi$ values

2010.

[31] J. Sahni and D. P. Vidyarthi, "A cost-effective deadline-constrained dynamic scheduling algorithm for scientific workflows in a cloud environment," IEEE Transactions on Cloud Computing, vol. 6, no. 1, pp. 2-18, 2015.

[32] M. Mao and M. Humphrey, "A performance study on the vm startup time in the cloud," in IEEE 5th International Conference on Cloud Computing, pp. 423-430, IEEE, 2012.

Panagiotis Oikonomou Dr. Panagiotis Oikonomou received his Diploma degree (2008) and M.Sc. degree (2010) from the Dept. of Electrical and Computer Engineering, University of Thessaly, Greece. 


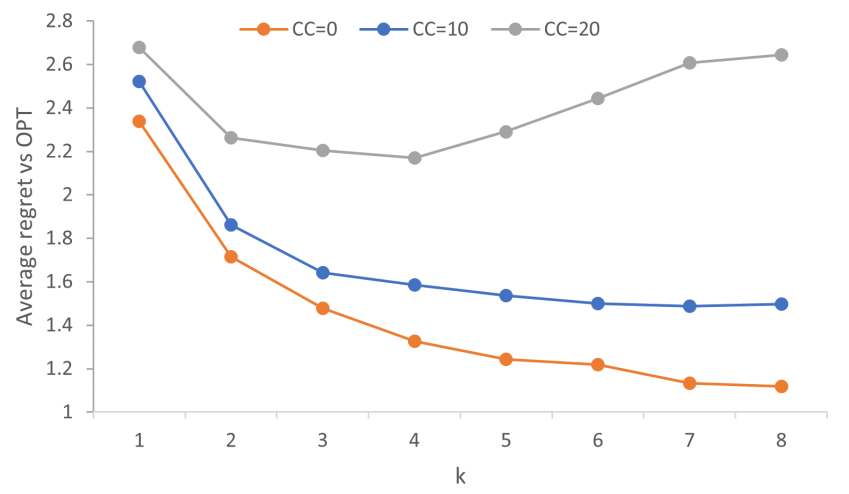

Fig. 13: Regret $\rho$ for different $k$ values (EPIGENOMICS)

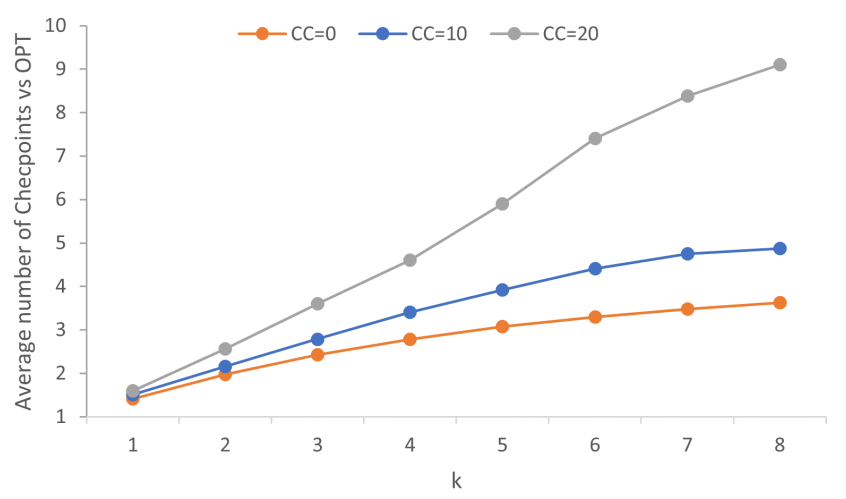

Fig. 14: $C T$ for different $k$ values (EPIGENOMICS)

He receives his $\mathrm{PhD}$ from the same dept in 2017. Currently he is a Lecturer in the Dept. of Computer Sciences, Univ. of Thessaly as a scientific scholarship holder. His current research interests include: CAD algorithms, Placement algorithms, Optimization problems, Heuristic algorithms, Soft Computational Methods, Fuzzy Logic and Desicion Support Systems. He is a member of groups developing open source platforms and member of Technical Chamber of Greece.

Kostas Kolomvatsos Dr Kostas Kolomvatsos received his B.Sc. in Informatics from the Department of Informatics at the Athens University of Economics and Business, his M.Sc. and his Ph.D. in Computer Science from the Department of Informatics and Telecommunications at the National and Kapodistrian University of Athens. Currently, he serves as an Assistant Professor in the Department of Informatics and Telecommunications, University of Thessaly. He was a Marie Skłodowska Curie Fellow (Individual Fellowship) at the School of Computing Science, University of Glasgow. His research interests are in the definition of Intelligent Systems adopting Machine Learning, Computational Intelligence and Soft Computing for Pervasive Computing, Distributed Systems, Internet of Things, Edge Computing and the management of Large Scale Data. $\mathrm{He}$ is the author of over 110 publications in the aforementioned areas.

Christos Anagnostopoulos Dr Christos Anagnostopoulos is an Associate Professor in the School of Computing Science, University of Glasgow. His expertise is in the areas of large-scale distributed data systems and in-network information processing. He has received funding for his research by the EC/H2020, UK EPSRC and the industry. Dr Anagnostopoulos is an author of over 150 refereed scientific journals/conferences. He is leading the Essence: Pervasive \& Distributed Intelligence within the Knowledge and Data Engineering Systems Group (IDA Section). He has held postdoctoral positions at University of Glasgow and University of Athens in the area of mobile and context-aware computing. He holds a BSc, MSc, and PhD in Computing Science, University of Athens (2008). He is an associate fellow of the HEA, member of the ACM and the IEEE. 